\title{
Regulação Emocional, Sintomas de Ansiedade e Depressão em Mulheres com Histórico de Violência Conjugal
}

\author{
Natália Zancan ${ }^{1}$ \\ Luisa Fernanda Habigzang ${ }^{1}$ \\ ${ }^{1}$ Pontifícia Universidade Católica do Rio Grande do Sul, Porto Alegre, RS
}

\begin{abstract}
Resumo
Este estudo avaliou as relações entre níveis de depressão, ansiedade e regulação emocional em 47 mulheres com experiência de violência conjugal. Também, foi investigada a gravidade das situações de violência e os níveis das agressões sofridas e perpetradas pelas mulheres. Por fim, foi investigado se há diferenças nos sintomas de depressão, ansiedade e regulação emocional nas mulheres que relataram sofrer violência e aquelas que identificaram sofrer e perpetrar violência. Os resultados indicaram sintomas leves de ansiedade e moderados de depressão e de desregulação emocional entre as participantes, e foram verificadas correlações positivas significativas entre tais sintomas. A comparação entre os grupos de mulheres que sofreram violência e aquelas que sofreram e perpetraram violência não indicou diferença nos níveis de depressão, ansiedade e regulação emocional. Tais resultados evidenciam que a exposição à violência conjugal representa um fator de risco para a saúde mental das mulheres e que a avaliação psicológica é útil para o desenvolvimento de estratégias de intervenção ajustadas às demandas clínicas.

Palavras-chave: violência conjugal, gênero, regulação emocional, avaliação psicológica
\end{abstract}

Emotional regulation, symptoms of anxiety and depression in women with a history of intimate partner violence

\begin{abstract}
This study evaluated depression, anxiety and emotional regulation levels in 47 women who experienced conjugal violence. Furthermore, it was investigated the severity of violence situations and the level of aggression suffered and perpetrated by women. Finally, we investigated possible differences in levels of depression, anxiety and emotional regulation between women who had reported suffering violence and those who reported suffering and perpetrating violence. The results indicated mild levels of anxiety, moderate levels of depression and emotional dysregulation among participants, showing significant correlations. The comparison between the group of women who had reported suffering violence and those who had suffered and perpetrated violence did not indicate differences in depression, anxiety and emotional regulation levels. These results show that exposure to intimate partner violence represents a risk factor for women's mental health and that psychological evaluation is useful for the development of intervention strategies adjusted to clinical demands.

Keywords: intimate partner violence; gender; emotional regulation; psychological evaluation

Regulación emocional, síntomas de ansiedad y depresión en mujeres con historia de violencia conjugal

\section{Resumen}

Este estudio evaluó las relaciones entre niveles de depresión, ansiedad y regulación emocional en 47 mujeres con experiencia de violencia conjugal. También fue investigada la gravedad de las situaciones de violencia y los niveles de las agresiones sufridas y cometidas por las mujeres. Por último, se investigó si hay diferencias en los síntomas de depresión, ansiedad y regulación emocional en las mujeres que relataron sufrir violencia y aquellas que identificaron sufrir y perpetrar violencia. Los resultados indicaron síntomas leves de ansiedad y moderados de depresión y de desregulación emocional entre las participantes, y se verificaron correlaciones positivas significativas entre tales síntomas. La comparación entre los grupos de mujeres que sufrieron violencia y las que sufrieron y perpetraron violencia no indicó diferencia en los niveles de depresión, ansiedad y regulación emocional. Tales resultados muestran que la exposición a violencia conjugal representa un factor de riesgo para la salud mental de las mujeres y que la evaluación psicológica es útil para el desarrollo de estrategias de intervención ajustadas a las demandas clínicas. Palabras-clave: violencia conjugal; género, regulación emocional; evaluación psicológica.
\end{abstract}

\section{Introdução}

A violência contra a mulher tem sido foco de pesquisas e intervenções por ser considerada um problema jurídico, social e de saúde pública (Lamoglia \& Minayo, 2009; Waiselfisz, 2015). Um ato de violência pode ser compreendido como uma ameaça à vida e vem acompanhado, com frequência, pelo silêncio da mulher, devido a complexa dinâmica que envolve aspectos individuais, familiares e sociais (Minayo,
2005). No Brasil, por muito tempo, a violência contra a mulher não foi considerada como um ato suscetível de punição. A pressão dos movimentos feministas foi fundamental para que a vitimização de mulheres pelos seus parceiros íntimos passasse a ter visibilidade social, tornando-se um problema público e não privado. A partir dos anos 80 , as demandas sociais para atenção à violência contra as mulheres contribuíram para a criação de casas de abrigo, de delegacias especializadas às mulheres e de centros de referência multiprofissionais 
com abordagem na violência física e sexual praticada pelo parceiro ou ex-parceiro conjugal. Deste modo, começou a se pensar em prevenção e tratamento para as mulheres em situação de violência (Gomes, Minayo, \& Silva, 2005; Schraiber \& D'Oliveira, 1999; Schraiber, D’Oliveira, Falcão, \& Figueiredo, 2005).

Em 2006, foi promulgada no Brasil a Lei no 11.340 (Lei Maria da Penha) com o objetivo de estabelecer estratégias e mecanismos para a prevenção e combate à violência contra a mulher (Garcia, Freitas, \& Hofelmann, 2013). Essa Lei define a violência doméstica e familiar contra a mulher como qualquer ato ou omissão, baseado em gênero, que cause lesão, sofrimento físico, sexual, psicológico, dano patrimonial, moral e, até mesmo, morte (Lei Federal no 11.340, 2006).

A violência não deve ser compreendida apenas como um problema jurídico e de segurança. Recentemente, a violência passou a ser considerada um problema de saúde pública, devido aos altos índices de incidência e às consequências negativas para a saúde física e mental das vítimas (Schraiber \& D'Oliveira, 1999). A Organização Mundial da Saúde (OMS, 2002), ao publicar o Relatório Mundial sobre Violência e Saúde, definiu a violência como o uso intencional de força ou de poder físico, de fato ou como ameaça, contra si mesmo, outra pessoa, grupo ou comunidade, que cause ou tenha probabilidade de causar lesões, morte, danos psicológicos, transtornos de desenvolvimento ou privações. A violência contra a mulher não se limita exclusivamente em danos lesivos de ordem física, engloba qualquer ação de dominação que a enfraqueça e a impossibilite de expressar seus propósitos enquanto pessoa (Tavares \& Pereira, 2007).

As agressões contra mulheres manifestam-se em diferentes contextos, dentre eles destacam-se aquelas cometidas no ambiente doméstico e familiar, sendo recorrentemente causada pelos seus parceiros íntimos (Santi, Nakano, \& Lettiere, 2010). Essas agressões ocorrem com maior frequência do que as cometidas por algum conhecido ou por um desconhecido (OMS, 2002).

Dentre os fatores de risco para ocorrência de violência contra mulher, destacam-se fatores individuais, tais como a experiência de violência na infância, tanto como vítima direta ou testemunha de agressão entre os pais, e fatores relacionais, tais como dificuldades de comunicação e de resolução de conflitos (Siegel, 2013; Stith \& McCollum, 2011). Fatores culturais também estão fortemente relacionados com a violência. A necessidade de poder, controle e maior força física em relação à mulher, bem como estereótipos baseados na desigualdade de gênero, contribuem para que os homens adotem papel de dominação nas relações e de autor de violência (Caldwell, Swan, \& Woodbrown, 2012; Lamoglia \& Minayo, 2009).

Muitos casos de violência contra mulheres no contexto doméstico não são notificados e a falta de informações oficiais impede a realização de relatórios precisos (OMS, 2002; Waiselfisz, 2015). Contudo, dados epidemiológicos indicam que um em cada cinco dias de absenteísmo no trabalho feminino é resultante da violência doméstica. Nos Estados Unidos, um terço das internações de mulheres em unidades de emergência ocorre devido às agressões sofridas em casa e, na América Latina, a violência doméstica acomete 25 a $50 \%$ das mulheres (Adeodato, Carvalho, Siqueira, \& Souza, 2005). Estimativas globais sobre a violência de gênero sugerem que 30\% das mulheres que tiveram um parceiro íntimo já sofreram violência física e/ ou sexual em algum momento da vida (OMS, 2014). No Brasil, estima-se que a cada quatro minutos uma mulher é agredida (D’Oliveira et al., 2009) e que entre $26 \%$ e $34,5 \%$ das mulheres sofrem violência física ou sexual por parte de seus parceiros (Vieira, Perdona, \& Santos, 2011).

As taxas de homicídios femininos referentes aos anos de 2003 a 2013, registradas pela OMS, apontam que o Brasil ocupa a quinta posição em meio a 83 países. Efetivamente, três países latino-americanos (El Salvador, Colômbia, Guatemala) e a Federação Russa demonstram índices superiores aos do Brasil. No ano de 2014, foram registrados pelo Sistema de Informação de Agravos de Notificação (SINAN) 223.796 atendimentos a mulheres que sofreram violência doméstica, sexual e/ou outras violências. O cônjuge ou ex-cônjuge foram os principais agressores (Waiselfisz, 2015).

\section{Violência Conjugal, Regulação Emocional, Depressão e Ansie- dade: Estudos Nacionais e Internacionais}

A violência é considerada um fator de risco para o desenvolvimento de doenças. Além de provocar lesões físicas, há o risco de desencadear prejuízos à saúde como dores crônicas, ansiedade, depressão, transtorno do estresse pós-traumático (TEPT), transtornos alimentares, entre outros. Ainda, pode ser um fator de risco para o consumo de bebidas alcoólicas e outras drogas (Adeodato et al., 2005; Deeke, Boing, Oliveira, \& Coelho, 2009; Dillon, Hussain, Loxton, \& Rahman, 2013; Hatzenberger, Lima, Lobo, Leite, \& Kristensen, 
2010; Zaleski, Pinsky, Laranjeira, Ramisetty-Mikler, \& Caetano, 2010).

Os transtornos de humor e de ansiedade são comumente identificados em mulheres com histórico de violência. Adeodato et al. (2005) avaliaram a qualidade de vida e o grau de depressão de mulheres vítimas da violência doméstica em uma amostra constituída por 100 mulheres que sofreram agressão de seus parceiros e que prestaram queixa na Delegacia da Mulher do Ceará. Foi observado que $72 \%$ das mulheres apresentaram sintomatologia clínica de depressão, com níveis moderados ou graves, com sentimentos de culpa em decorrência das agressões, insônia, ideação suicida e $78 \%$ tinham sintomas de ansiedade. O estudo de Mozzambani, Ribeiro, Fuso, Fiks e Mello (2012) avaliou a presença de sintomas psicopatológicos em 17 mulheres em situação de violência doméstica, e os resultados indicaram que $89 \%$ da amostra apresentava sintomatologia clínica para depressão e $94 \%$ para ansiedade.

Outro estudo avaliou a eficácia de um programa de intervenção psicológica para mulheres que foram maltratadas pelo parceiro íntimo. A amostra foi constituída por 128 mulheres usuárias de serviços públicos especializados. Noventa e seis mulheres participaram do programa de intervenção e 32 fizeram parte de um grupo controle. Inicialmente, foram avaliados os níveis de depressão e ansiedade, sintomas de estresse póstraumático, e níveis de autoestima. $\mathrm{Na}$ avaliação inicial, $8,4 \%$ das participantes da intervenção apresentavam depressão mínima, 10,5\% leve, 33,7\% moderada e $47,4 \%$ da amostra apresentava níveis graves de depressão. Em relação aos níveis de ansiedade, $23,5 \%$ da amostra apresentava níveis leves, $11,8 \%$ níveis moderados e $64,7 \%$ ansiedade grave. Além disso, 49,5\% da amostra apresentavam sintomas de estresse pós-traumático. Verificou-se que, após a intervenção psicológica, as mulheres reduziram significativamente os sintomas de depressão, de ansiedade e os sintomas de estresse póstraumático (Matud, Fortes, \& Medina, 2014).

A depressão é um transtorno caracterizado pela presença de humor deprimido quase todos os dias. Os sintomas podem incluir sentimento de culpa e inutilidade, capacidade diminuída para se concentrar, pensamentos recorrentes de morte, insônia, alterações no apetite, alterações psicomotoras, fadiga, queixas somáticas e perda de interesse pelas atividades. Estes sintomas devem estar presentes durante pelo menos duas semanas. Por sua vez, os transtornos de ansiedade incluem transtornos que compartilham características de medo e ansiedade excessivos, bem como alterações de comportamento. Verificam-se comportamentos de vigilância, associados a tensão muscular. Além disso, são comuns alterações fisiológicas, tais como sudorese, tremores, taquicardia, sensação de tontura e asfixia. Por fim, preocupações excessivas (apreensão) com diferentes eventos ou atividades estão presentes (American Psychiatric Association, 2014).

Em decorrência da sintomatologia clínica associada à violência, pesquisas apontam que as mulheres fazem uso de ansiolíticos ou antidepressivos para suportar o sofrimento psicológico (Adeodato et al., 2005; Deeke et al., 2009). O estudo de Deeke et al. (2009) realizado com 30 casais para avaliar a dinâmica da violência doméstica a partir do discurso da mulher agredida e do parceiro autor da agressão, apontou que $56,7 \%$ das mulheres da pesquisa faziam uso de medicamentos em função da situação de violência. De acordo com os autores, as mulheres podem perceber os efeitos da medicação como estratégia para resolver problemas, conflitos e tolerar emoções negativas.

Outra consequência em decorrência à exposição à violência é o prejuízo na regulação emocional (Siegel \& Forero, 2012). A regulação emocional pode ser definida como a capacidade de manter, aumentar ou diminuir os componentes de uma resposta emocional. Esse processo abrange a consciência e o entendimento das emoções, incluindo seus componentes fisiológicos e comportamentais, além da habilidade de controlar comportamentos impulsivos e de comportar-se de forma funcional ao experienciar emoções negativas (Gratz \& Roemer, 2004). Trata-se de um processo baseado no manejo das emoções, atrelado à capacidade de modulá-las quanto ao tempo de duração e sua intensidade (Gross, 2013; Rodrigues \& Gondim, 2013). A violência pode afetar negativamente a identificação, expressão e manejo das emoções (Zamir \& Lavee, 2014). Tais dificuldades podem prejudicar relações interpessoais e estar associadas a sintomas de depressão (Berzenski \& Yates, 2010) e ansiedade (Siegel \& Forero, 2012).

Pesquisas apontam a relação entre exposição a maus tratos na infância e a desregulação emocional na idade adulta (Gratz, Paulson, Jakupcak, \& Tull, 2009; Siegel \& Forero, 2012; Zamir \& Lavee, 2014). Mulheres em situação de violência conjugal comumente apresentam histórico de maus tratos na infância e a exposição prolongada à violência pode agravar dificuldades de regulação emocional (Berzenski \& Yates, 2010).

A relação entre desregulação emocional, múltiplas formas de abuso infantil e estresse pós-traumático subsequente foi avaliada por Burns, Jackson e Harding 
(2011) em 912 mulheres. Os resultados indicaram que as mulheres que reportaram uma história de abuso sexual, físico ou emocional apresentaram maiores níveis de desregulação emocional em comparação com as mulheres sem histórico de abusos. Os autores também identificaram que o abuso emocional na infância foi preditor da desregulação emocional.

Outro estudo investigou se as relações entre abuso na infância, violência conjugal e qualidade no relacionamento são moderadas pela capacidade de regulação das emoções. A amostra foi composta por 425 mulheres casadas ou em união estável. Os resultados demonstraram que o abuso na infância esteve associado a altos níveis de violência conjugal. Além disso, foi verificado que o abuso na infância teve efeito negativo na qualidade do relacionamento de mulheres que apresentavam baixos níveis de regulação emocional em comparação com as mulheres com um alto nível de regulação emocional. Desse modo, os autores referem que a regulação emocional é um fator de proteção contra a revitimização em relacionamentos íntimos (Zamir \& Lavee, 2014).

A violência conjugal comumente é compreendida como uma forma de violência contra a mulher (Castro \& Riquer, 2003). Contudo, em uma relação conjugal, a mulher também pode apresentar comportamentos agressivos (Krug, Dahlberg, Mercy, Zwi, \& Lozano, 2002). Estes comportamentos podem ser estratégias de resistência, defesa ou resposta ao ciclo de violência. Assim, é necessário investigar a ocorrência de violência mútua nas relações íntimas com objetivo de compreender as dinâmicas relacionais neste fenômeno (Zaleski, Pinsky, Laranjeira, Ramisetty-Mikler, \& Caetano, 2010). A perspectiva relacional da violência não deve ter como objetivo minimizar ou justificar agravos da violência contra a mulher, pois em função de desigualdades de gênero, as mulheres estão em situação de desvantagem em nossa sociedade.

Nesta perspectiva, para estimar a prevalência de violência por parceiro íntimo, Zaleski et al. (2010) entrevistaram 814 mulheres e 631 homens. Os resultados indicaram que as mulheres apresentaram prevalência de 14,6\% de episódios de violência por parceiros e os homens de $10,7 \%$. O tipo de violência prevalente, considerado leve, foi empurrar, agarrar ou sacudir, sendo $7,4 \%$ perpetrado por homens e $9,3 \%$ por mulheres. $\mathrm{O}$ tipo mais comum de violência relatado foi a agressão por meio de tapas. Além disso, os atos de violência grave ocorreram em menor frequência do que os de violência leve. Estes resultados indicaram comportamentos mútuos de agressão entre parceiros.
A partir desses dados, verifica-se a necessidade de fomentar estudos empíricos que avaliem o impacto da violência conjugal para a saúde mental das mulheres. Além disso, investigar níveis de regulação emocional pode contribuir para entender a dinâmica de relações conjugais violentas. O desenvolvimento de pesquisas sobre avaliação psicológica de mulheres em situação de violência responde a uma demanda social e de saúde pública, uma vez que pode contribuir para elaboração de planos preventivos e terapêuticos efetivos.

Considerando o potencial impacto negativo da violência, este estudo teve como objetivo investigar níveis de depressão, ansiedade e regulação emocional em mulheres com histórico de violência conjugal, bem como relações entre estes sintomas. Além disso, buscou verificar os tipos de agressões sofridas e perpetradas pelas mulheres. Por fim, foi analisado se há diferenças nos níveis de depressão, ansiedade e regulação emocional nas mulheres que relataram sofrer violência e naquelas que identificaram sofrer e perpetrar violência no contexto conjugal.

\section{Método}

\section{Delineamento}

Estudo quantitativo, correlacional e transversal (Robson, 1993).

\section{Participantes}

Participaram do estudo 47 mulheres com histórico de violência conjugal que não apresentavam sintomas psicóticos e comprometimento cognitivo grave evidente, avaliados por meio de entrevista e observação clínica. A amostra foi não probabilística e as mulheres tinham idade entre 19 e 57 anos $(M=32,11$ anos; $D P$ $=10,70$ anos), sendo que $19 \%$ declararam serem solteiras, $28 \%$ casadas, $17 \%$ divorciadas e $36 \%$ definiram manter outras formas de relacionamento. O tempo de relacionamento médio foi de seis anos $(D P=7$ anos; amplitude de 2 meses a 26 anos). No total da amostra, $60 \%$ possuíam ao menos um filho e $40 \%$ não tinham filhos. Entre as participantes que possuíam filhos, 38\% tinham um filho, $31 \%$ possuíam dois filhos, $23 \%$ tinham três filhos e $8 \%$ tinham quatro filhos. Em relação a escolaridade, 9\% não concluíram o ensino fundamental, $2 \%$ possuíam o ensino fundamental completo, $13 \%$ não cursaram o ensino médio completo, 19\% havia concluído o ensino médio, $23 \%$ possuíam ensino superior incompleto e $34 \%$ havia cursado algum curso de ensino superior. Quanto à situação laboral, 73\% das 
participantes estavam trabalhando no momento da entrevista e $27 \%$ não estavam. Foi observado que $42 \%$ das participantes fazia uso de psicofármacos.

\section{Instrumentos}

1. Questionário de Dados Sociodemográficos (Lima, 2010). Investigou características como idade, estado civil, escolaridade, situação laboral, número de filhos. Além disso, foi investigado se as participantes ou seus familiares apresentavam algum problema de saúde, e se usavam medicações ou substâncias ilícitas.

2. Inventário de Ansiedade Beck - BAI (Beck Anxiety Inventory - BAI). Foi desenvolvido por Beck, Epstein, Brown e Sterr em 1998, e adaptado e validado para o Brasil por Cunha (2001). Objetivou investigar a intensidade dos sintomas de ansiedade por meio de uma escala de 21 itens, em que o escore total é a soma das pontuações de cada item (0 a 63), com os seguintes pontos de corte: 0-10 para nível mínimo de ansiedade, 11-19 para nível leve, 20-30 para nível moderado e 31-63 para nível grave. A escala original apresenta uma estrutura unifatorial, com alfa de Cronbach satisfatório $(\alpha=0,92)$ (Cunha, 2001). No presente estudo o índice de consistência interna da escala foi adequado $(\alpha=0,91)$

3. Inventário de Depressão Beck - BDI-II (Beck Depression Inventory - BDI-II). Desenvolvido por Beck, Steer e Brown em 1996, adaptado e validado para o Brasil por Gorenstein, Pang, Argimon e Werlang (2011). Avaliou os sintomas de depressão por meio de uma escala autoaplicável composta por 21 itens em que o escore total é a soma da pontuação de cada item ( 0 a 63), com os seguintes pontos de corte: 0-13 para depressão mínima, 14-19 para depressão leve a, 20-28 para depressão moderada e 29-63 para depressão grave. A versão original da escala apresenta coeficiente alfa de Cronbach $(\alpha$ $=0,85$ ) (Gorenstein, Pang, Argimon, \& Werlang, 2011). Na amostra investigada o coeficiente alpha de Cronbach da escala foi satisfatório $(\alpha=0,90)$.

4. Escala Tática de Conflito Revisada (Revised Conflict Tatic Scale-CTS2). Desenvolvida por Straus et al. (1996) e traduzida e adaptada para o Brasil por Moraes, Hasselman e Reichenheim (2002). Avalia a violência nas relações amorosas por meio de cinco dimensões: (1) agressão física, (2) agressão psicológica, (3) coerção sexual, (4) lesão corporal, e (5) negociação. É composta por 39 itens agrupados em pares de perguntas destinados a violência sofrida e perpetrada, totalizando 78 itens. $\mathrm{O}$ valor do alfa de Cronbach referente à escala total para a perpetração é $\alpha=0,79$ e para a vitimização $\alpha=0,80$ (Moraes et al., 2002). Na amostra investigada foram verificados os valores de consistência interna para as dimensões negociação (perpetrador, $\alpha=0,82$; vítima, $\alpha=0,62$ ), agressão psicológica (perpetrador, $\alpha=0,77$; vítima, $\alpha=0,73$ ), agressão física (perpetrador, $\alpha=0,86$; vítima, $\alpha=0,75)$, lesão corporal (perpetrador, $\alpha=0,63$; vítima, $\alpha=0,61$ ) e coerção sexual (perpetrador, $\alpha=0,83$; vítima, $\alpha=0,89$ ).

5. Escala de Dificuldades de Regulação Emocional - DERS (Difficulties in Emotion Regulation Scale - DERS). Desenvolvida em 2004 por Gratz e Roemer e validada para uma amostra portuguesa por Coutinho, Ribeiro, Ferreirinha e Dias (2009). Avaliou os níveis de desregulação em seis domínios: não aceitação das emoções negativas $(\alpha=0,86)$; capacidade para controlar comportamentos impulsivos e agir de acordo com os objetivos desejados em momentos de emoção negativa $(\alpha=0,85)$; dificuldades em controlar comportamento impulsivo quando experiencia emoções negativas $(\alpha=0,80)$; acesso limitado a estratégias de regulação emocional que são percebidas como efetivas $(\alpha=0,88)$; falta de consciência emocional $(\alpha=0,74)$; e falta de clareza emocional $(\alpha=0,75)$. Contêm 36 itens numa escala de cinco pontos, sendo 1 (quase nunca se aplica a mim) a 5 (aplica-se quase sempre a mim). $\mathrm{Na}$ amostra de Coutinho et al., (2009), o alfa de Cronbach foi $\alpha=0,924$, e $\alpha \geq 0,75$ para cada subescala. Na presente pesquisa, foi utilizada a versão de adaptação semântica para o Brasil, desenvolvida por Boeckel (2013). Essa versão apresenta alfa de Cronbach de 0,85 . No presente estudo, os valores de alfa de Cronbach da escala e suas subdimensões foram satisfatórios (regulação emocional, $\alpha=0,86$; não aceitação das emoções negativas, $\alpha=0,81$; capacidade para controlar comportamentos impulsivos e agir de acordo com os objetivos desejados em momentos de emoção negativa, $\alpha=0,91$; dificuldades em controlar comportamento impulsivo quando experiencia emoções negativas, $\alpha=0,75$; acesso limitado a estratégias de regulação emocional que são percebidas como efetivas, $\alpha=0,72$; falta de consciência emocional, $\alpha=0,63$; e falta de clareza emocional, $\alpha=0,72$ ). 


\section{Procedimentos Éticos e de Coleta de Dados}

O projeto foi aprovado pelo Comitê de Ética em Pesquisa (CEP) da Pontifícia Universidade Católica do Rio Grande do Sul, sob parecer no 1.000.590. Após a aprovação, foi realizado o treinamento da equipe para coleta de dados, por meio de seminários teóricos sobre o tema e para aplicação dos instrumentos. A coleta foi realizada por psicólogas e estudantes do curso psicologia na Casa de Apoio à Mulher Vítima da Violência na cidade de Passo Fundo/RS e no Serviço de Atendimento da Universidade Serviço de Atendimento da Universidade em Porto Alegre/RS. As mulheres foram convidadas a participar da pesquisa após o atendimento inicial prestado pelos locais. Inicialmente foi apresentado o Termo de Consentimento Livre e Esclarecido (TCLE), contendo informações sobre os propósitos da pesquisa e assegurando-lhes o sigilo da identidade. O TCLE está de acordo com a resolução 466/12 do Ministério da Saúde (2012). Após a concordância em participar da pesquisa foi realizada a aplicação dos instrumentos. O questionário de dados pessoais e sociodemográficos foi aplicado em forma de entrevista e os demais instrumentos foram aplicados em ordem aleatória para evitar efeito de ordem.

\section{Análise dos Dados}

Foram realizadas análises descritivas das participantes para conhecer características sociodemográficas. As médias e desvios padrão de cada instrumento foram avaliados por meio de estatísticas descritivas. A fim de avaliar as relações entre as características das participantes (idade e escolaridade), com as dimensões de regulação emocional, níveis dos tipos de agressão conjugal para a vitimização e perpetração (negociação, agressão psicológica, agressão física, lesão corporal e coerção sexual), níveis de depressão e ansiedade foram realizadas análises de correlação de rho de Spearman. Além disso, foram desenvolvidas correlações Ponto Bisseriais com as variáveis citadas e o uso de psicofármacos pelas participantes. As correlações Ponto Bisseriais foram desenvolvidas a fim de investigar as associações do uso de psicofármacos (sim e não), variável categórica, com as outras variáveis investigadas no estudo. Foi investigado se havia diferenças nas dimensões de regulação emocional e nos níveis de depressão e ansiedade comparando o grupo de mulheres que identificaram ter sofrido agressões e o grupo de mulheres que identificaram ter sofrido e perpetrado agressões. Tal comparação de grupos foi realizada por meio do teste Kolmogorov-Smirnov Z. O grupo de mulheres que sofreu agressões e o grupo de mulheres que sofreu e perpetrou agressões foram definidos com base na média das participantes nas dimensões de táticas de resolução de conflitos, adotada para este estudo como ponto de corte.

As análises foram desenvolvidas por meio de testes não-paramétricos, pois a avaliação da normalidade de distribuição, verificada pelos testes Kolmogorov-Smirnov e Shapiro-Wilk, indicou a não-normalidade dos dados $(\phi<0,05)$. Em razão do tamanho da amostra, foi utilizado o exact probability test (Monte Carlo) nas análises de comparação de grupos (Kolmogorov-Smirnov Z), uma vez que o uso deste método aliado aos testes não-paramétricos apresenta maior acurácia (Field, 2005).

\section{Resultados}

As análises descritivas indicaram que a média dos níveis de ansiedade $(M=17,0 ; D P=11,2)$ e depressão $(M=23,8 ; D P=11,2)$ foram leve e moderado, respectivamente (Cunha, 2001). Em relação às dimensões de regulação emocional, compreende-se que altos índices indicam a presença de dificuldades de regulação emocional. Foi observado que as participantes apresentavam índices moderados no escore total de regulação emocional $(M=109,6 ; D P=20,7)$ e nas subescalas avaliadas (não aceitação das emoções negativas, $M=17,6$, $D P=7,2$; capacidade para controlar comportamentos impulsivos e agir de acordo com os comportamentos desejados em momentos de emoções negativas, $M=$ 22,6, $D P=4,6$; dificuldades em controlar comportamento impulsivo quando vivencia emoções negativas, $M=14,8, D P=5,1$; acesso limitado a estratégias de regulação emocional que são percebidas como efetivas, $M=14,8, D P=5,1$; falta de consciência emocional, $M=16,2, D P=4,4$; e falta de clareza emocional, $M$ $=14,6, D P=2,7)$, indicando dificuldades na regulação emocional. A média das participantes nas dimensões de vitimização (negociação, $M=3,8, D P=2,0$; agressão psicológica, $M=5,1, D P=1,3$; agressão física, $M=6,1$, $D P=3,0$; lesão corporal, $M=1,0, D P=0,8$; e coerção sexual, $M=1,4, D P=2,3$ ) e perpetração (negociação, $M=5,3, D P=1,0$; agressão psicológica, $M=3,9, D P=$ 2,0; agressão física, $M=2,6, D P=2,3$; lesão corporal, $M=2,6, D P=1,7$; e coerção sexual, $M=0,1, D P=0,3$ ) apresentaram valores entre baixos e moderados.

Entre as características sociodemográficas, apenas a idade esteve positivamente relacionada ao uso de psicofármacos. A idade, escolaridade e o uso de psicofármacos não estiveram associados aos níveis 
de ansiedade, depressão e aos índices de regulação emocional e suas dimensões. Os níveis de ansiedade e depressão apresentaram correlação positiva. Os níveis de ansiedade estiveram positivamente relacionados com todas as dimensões que compõem a regulação emocional, exceto a falta de consciência emocional, com a qual apresentou correlação negativa. Os níveis de depressão estiveram positivamente relacionados às dimensões não aceitação das emoções negativas, dificuldades em controlar comportamento impulsivo quando vivencia emoções negativas, acesso limitado a estratégias de regulação emocional que são percebidas como efetivas e falta de clareza emocional. No entanto, os níveis de depressão apresentaram uma relação negativa com a dimensão falta de consciência emocional e não estiveram relacionados à dimensão capacidade para controlar comportamentos impulsivos e agir de acordo com os objetivos desejados em momentos de emoções negativas. As correlações entre ansiedade, depressão e regulação emocional estão apresentadas na Tabela 1.

A vitimização por agressão psicológica esteve positivamente associada à dimensão capacidade para

Tabela 1

Correlações entre Idade, Escolaridade, Psicofármacos, Dimensões de Regulação Emocional, Índices de Vitimização, Perpetração, Depressão, Ansiedade

\begin{tabular}{|c|c|c|c|c|c|c|c|c|c|c|}
\hline & 1 & 2 & 3 & 4 & 5 & 6 & 7 & 8 & 9 & 10 \\
\hline \multicolumn{11}{|l|}{ 1) $I$} \\
\hline 2) Es & $0,19^{a}$ & & & & & & & & & \\
\hline 3) UP & $0,51^{b * *}$ & $0,20^{b}$ & & & & & & & & \\
\hline 4) $\mathrm{A}$ & $-0,01^{a}$ & $0,21^{a}$ & $-0,05^{b}$ & & & & & & & \\
\hline 5) $\mathrm{D}$ & $0,03^{a}$ & $0,02^{a}$ & $0,03^{b}$ & $0,50^{a * *}$ & & & & & & \\
\hline 6) LE & $-0,13^{a}$ & $0,01^{a}$ & $-0,11^{b}$ & $0,39^{a * *}$ & $0,65^{a * *}$ & & & & & \\
\hline 7) $\mathrm{NE}$ & $-0,09^{a}$ & $0,10^{a}$ & $-0,09^{b}$ & $0,55^{a^{* *}}$ & $0,57^{a^{* *}}$ & $0,66^{a^{* *}}$ & & & & \\
\hline 8) FC & $0,02^{a}$ & $0,10^{a}$ & $-0,13^{b}$ & $-0,42^{a^{* *}}$ & $-0,42^{a^{* *}}$ & $-0,36^{a^{*}}$ & $-0,36^{a^{*}}$ & & & \\
\hline 9) $\mathrm{CI}$ & $-0,09^{a}$ & $0,26^{a}$ & $-0,07^{b}$ & $0,34^{a^{*}}$ & $0,28^{a}$ & $0,57^{a * *}$ & $0,61^{a^{* *}}$ & $-0,10^{a}$ & & \\
\hline 10) DI & $0,10^{a}$ & $0,19^{a}$ & $0,14^{b}$ & $0,38^{a * *}$ & $0,54^{a * *}$ & $0,66^{a * *}$ & $0,54^{a^{* *}}$ & $-0,44^{a^{* *}}$ & $0,39^{a^{* *}}$ & \\
\hline 11) $\mathrm{CE}$ & $-0,05^{a}$ & $0,12^{a}$ & $-0,06^{b}$ & $0,16^{a}$ & $0,34^{a^{*}}$ & $0,39^{a * *}$ & $0,16^{a}$ & $-0,18^{a}$ & $0,13^{a}$ & $0,15^{a}$ \\
\hline 12) $R E$ & $-0,11^{a}$ & $0,18^{a}$ & $-0,10^{b}$ & $0,47^{a * *}$ & $0,59^{a * *}$ & $0,87^{a^{* *}}$ & $0,84^{a^{* *}}$ & $-0,22^{a}$ & $0,76^{a^{* *}}$ & $0,68^{a^{* *}}$ \\
\hline 13) $\mathrm{VN}$ & $-0,33^{a^{*}}$ & $0,08^{a}$ & $-0,017^{b}$ & $0,07^{a}$ & $0,03^{b}$ & $-0,15^{a}$ & $0,33^{a^{*}}$ & $0,07^{a}$ & $-0,04^{a}$ & $0,11^{a}$ \\
\hline 14) VAP & $0,24^{a}$ & $0,06^{a}$ & $-0,06^{b}$ & $-0,13^{a}$ & $0,05^{b}$ & $-0,08^{a}$ & $0,07^{a}$ & $-0,15^{a}$ & $0,29^{a^{*}}$ & $0,01^{a}$ \\
\hline 15) VAF & $0,16^{a}$ & $-0,3^{a^{*}}$ & $-0,08^{b}$ & $-0,01^{a}$ & $-0,03^{b}$ & $0,03^{a}$ & $0,03^{a}$ & $-0,06^{a}$ & $0,13^{a}$ & $0,01^{a}$ \\
\hline 15) VLC & $-0,13^{a}$ & $-0,13^{a}$ & $-0,07^{b}$ & $-0,19^{a}$ & $0,02^{b}$ & $0,14^{a}$ & $-0,03^{a}$ & $-0,13^{a}$ & $0,15^{a}$ & $-0,04^{a}$ \\
\hline 16) VCS & $0,44^{a * *}$ & $0,16^{a}$ & $0,11^{b}$ & $-0,24^{a}$ & $-0,13^{b}$ & $0,15^{a}$ & $0,04^{a}$ & $-0,32^{a^{*}}$ & $-0,11^{a}$ & $-0,17^{a}$ \\
\hline 18) PN & $0,01^{a}$ & $0,21^{a}$ & $0,08^{b}$ & $-0,05^{a}$ & $0,17^{b}$ & $-0,09^{a}$ & $0,31^{a^{*}}$ & $0,04^{a}$ & $-0,12^{a}$ & $0,08^{a}$ \\
\hline 19) PAP & $-0,02^{a}$ & $-0,10^{a}$ & $-0,01^{b}$ & 0,07 & $0,17^{b}$ & $-0,08^{a}$ & $0,34^{a^{*}}$ & $0,02^{a}$ & $0,08^{a}$ & $0,19^{a}$ \\
\hline 20) PAF & $-0,31^{a^{*}}$ & $-0,29^{a}$ & $-0,20^{b}$ & $0,04^{a}$ & $0,06^{b}$ & $0,12^{a}$ & $0,16^{a}$ & $-0,18^{a}$ & $0,01^{a}$ & $0,04^{a}$ \\
\hline 21) PLC & $0,43^{a * *}$ & $-0,31^{a^{*}}$ & $0,01^{b}$ & $-0,05^{a}$ & $-0,09^{b}$ & $0,02^{a}$ & $-0,11^{a}$ & $-0,04$ & $-0,02^{a}$ & $-0,07^{a}$ \\
\hline 22) PCS & $0,28^{a}$ & $0,11^{a}$ & $0,21^{b}$ & 0,17 & $0,26^{b}$ & $-0,04^{a}$ & $0,35^{a^{*}}$ & $0,23^{a}$ & $0,22^{a}$ & $0,34^{a^{*}}$ \\
\hline
\end{tabular}

Nota. ${ }^{*}-p \leq 0,05 ;{ }^{*}{ }_{-} p \leq 0,001 ; M$ - Média; DP - Desvio Padrão; $a$-Correlações de Spearman's rho; $b$ - Correlações Ponto Bisserial; I = Idade; Es = Escolaridade; UP = Uso de Psicofármacos; A = Ansiedade; $\mathrm{D}=$ Depressão $; \mathrm{LE}=$ Acesso Limitado a Estratégias de Regulação Emocional que são Percebidas como Efetivas; NE = Não Aceitação das Emoções Negativas; FC = Falta de Consciência Emocional; CI = Capacidade para Controlar Comportamentos Impulsivos; DI = Dificuldades em Controlar Comportamento Impulsivo quando Vivencia Emoções Negativas; CE = Falta de Clareza Emocional; RE = Regulação Emocional; VN = Vitimização - Negociação; VAP = Vitimização - Agressão Psicológica; VAF = Vitimização - Agressão Física; VLC = Vitimização - Lesão Corporal; VCS = Vitimização - Coerção Sexual; PN = Perpetração - Negociação; PAP = Perpetração - Agressão Psicológica; PAF = Perpetração - Agressão Física; PLC = Perpetração - Lesão Corporal; PCS = Perpetração Coerção Sexual. 
controlar comportamentos impulsivos. A dimensão que avalia vitimização por agressão física esteve negativamente relacionada com a idade e positivamente com a vitimização por agressão psicológica. A dimensão lesão corporal apresentou uma relação negativa com a dimensão negociação e positiva com agressão física. A presença de vitimização por coerção sexual esteve positivamente relacionada à idade e a vitimização por agressão física e negativamente à falta de consciência emocional (Tabela 1).

A dimensão agressão psicológica como perpetradora esteve positivamente relacionada a não aceitação das emoções negativas, aos índices de negociação, e agressão psicológica como vítima. A agressão física como perpetradora esteve negativamente relacionada com a idade e positivamente com coerção sexual como vítima e agressão psicológica como perpetradora. As ações de lesão corporal como perpetradora estiveram positivamente associadas à idade, a agressão psicológica e física como vítima e negativamente à escolaridade. A coerção sexual como perpetradora esteve positivamente associada a não aceitação das emoções negativas, dificuldades em controlar comportamento impulsivo quando vivencia emoções negativas e desregulação emocional (Tabela 1).

A amostra foi dividida em dois grupos para avaliar se as participantes que sofreram agressões (G1) diferenciavam-se das que sofreram e perpetraram agressões (G2) nos níveis de depressão, ansiedade, regulação emocional e suas dimensões. Os resultados estão apresentados na Tabela 2 .

Tabela 2

Diferenças dos Niveis de Regulação Emocional, Depressão, Ansiedade entre G1 e G2 nas Dimensões de Vitimização/Perpetração

\begin{tabular}{|c|c|c|c|c|c|c|c|c|c|c|}
\hline & \multicolumn{2}{|c|}{ Negociação } & \multicolumn{2}{|c|}{$\begin{array}{l}\text { Agressão } \\
\text { Psicológica }\end{array}$} & \multicolumn{2}{|c|}{ Agressão Física } & \multicolumn{2}{|c|}{ Lesão Corporal } & \multicolumn{2}{|c|}{ Coerção Sexual } \\
\hline & $\mathrm{V}$ & $\mathrm{V}-\mathrm{P}$ & $\mathrm{V}$ & V-P & $\mathrm{V}$ & $\mathrm{V}-\mathrm{P}$ & $\mathrm{V}$ & V-P & $\mathrm{V}$ & V-P \\
\hline & $M(D P)$ & $M(D P)$ & $M(D P)$ & $M(D P)$ & $M(D P)$ & $M(D P)$ & $M(D P)$ & $M(D P)$ & $M(D P)$ & $M(D P)$ \\
\hline $\mathrm{A}$ & $\begin{array}{l}17,7 \\
(13,4)\end{array}$ & $\begin{array}{l}16,3 \\
(8,9)\end{array}$ & $\begin{array}{l}17,5 \\
(13,1)\end{array}$ & $\begin{array}{l}16,6 \\
(9,5)\end{array}$ & $\begin{array}{l}18,1 \\
(11,3)\end{array}$ & $\begin{array}{l}6,0 \\
(11,3)\end{array}$ & $\begin{array}{l}15,8 \\
(9,8)\end{array}$ & $\begin{array}{l}18,2 \\
(12,7)\end{array}$ & $\begin{array}{l}16,7 \\
(11,5)\end{array}$ & $\begin{array}{l}21,2 \\
(8,7)\end{array}$ \\
\hline $\mathrm{D}$ & $\begin{array}{l}25,8 \\
(15,6)\end{array}$ & $\begin{array}{l}21,7 \\
(11,7)\end{array}$ & $\begin{array}{l}22,7 \\
(16,3)\end{array}$ & $\begin{array}{l}24,8 \\
(11,3)\end{array}$ & $\begin{array}{l}23,8 \\
(12,2)\end{array}$ & $\begin{array}{l}23,8 \\
(15,3)\end{array}$ & $\begin{array}{l}20,4 \\
(13,4)\end{array}$ & $\begin{array}{l}27,3 \\
(13,5)\end{array}$ & $\begin{array}{l}23,2 \\
(14,2)\end{array}$ & $\begin{array}{l}29,5 \\
(6,5)\end{array}$ \\
\hline LE & $\begin{array}{l}24,3 \\
(8,1)\end{array}$ & $\begin{array}{l}23,1 \\
(7,3)\end{array}$ & $\begin{array}{l}23,3 \\
(9,0)\end{array}$ & $\begin{array}{l}24,0 \\
(6,4)\end{array}$ & $\begin{array}{l}23,4 \\
(7,2)\end{array}$ & $\begin{array}{l}24,0 \\
(8,1)\end{array}$ & $\begin{array}{l}23,2 \\
(8,1)\end{array}$ & $\begin{array}{l}24,2 \\
(7,3)\end{array}$ & $\begin{array}{l}23,4 \\
(7,9)\end{array}$ & $\begin{array}{l}26,7 \\
(2,7)\end{array}$ \\
\hline $\mathrm{NE}$ & $\begin{array}{l}16,2 \\
(7,7)\end{array}$ & $\begin{array}{l}18,9 \\
(6,5)\end{array}$ & $\begin{array}{l}16,1 \\
(7,5)\end{array}$ & $\begin{array}{l}19,0 \\
(6,7)\end{array}$ & $\begin{array}{l}16,8 \\
(6,2)\end{array}$ & $\begin{array}{l}18,4 \\
(8,0)\end{array}$ & $\begin{array}{l}17,0 \\
(7,3)\end{array}$ & $\begin{array}{l}18,3 \\
(7,2)\end{array}$ & $\begin{array}{l}17,1 \\
(7,1)\end{array}$ & $\begin{array}{l}23,2 \\
(5,7)\end{array}$ \\
\hline $\mathrm{FC}$ & $\begin{array}{l}22,6 \\
(5,7)\end{array}$ & $\begin{array}{l}22,5 \\
(3,4)\end{array}$ & $\begin{array}{l}23,0 \\
(5,2)\end{array}$ & $\begin{array}{l}22,2 \\
(4,1)\end{array}$ & $\begin{array}{l}22,2 \\
(4,5)\end{array}$ & $\begin{array}{l}22,9 \\
(4,8)\end{array}$ & $\begin{array}{l}22,6 \\
(4,9)\end{array}$ & $\begin{array}{l}22,5 \\
(4,4)\end{array}$ & $\begin{array}{l}22,6 \\
(4,8)\end{array}$ & $\begin{array}{l}22,5 \\
(1,7)\end{array}$ \\
\hline CI & $\begin{array}{l}14,2 \\
(5,7)\end{array}$ & $\begin{array}{l}15,5 \\
(4,5)\end{array}$ & $\begin{array}{l}13,3 \\
(4,6)\end{array}$ & $\begin{array}{l}16,2 \\
(5,2)\end{array}$ & $\begin{array}{l}13,9 \\
(4,5)\end{array}$ & $\begin{array}{l}15,7 \\
(5,5)\end{array}$ & $\begin{array}{l}14,5 \\
(4,7)\end{array}$ & $\begin{array}{l}15,3 \\
(5,6)\end{array}$ & $\begin{array}{l}14,3 \\
(5,0)\end{array}$ & $\begin{array}{l}20,5 \\
(2,5)\end{array}$ \\
\hline DI & $\begin{array}{l}16,1 \\
(5,0)\end{array}$ & $\begin{array}{l}16,2 \\
(4,0)\end{array}$ & $\begin{array}{l}15,0 \\
(5,0)\end{array}$ & $\begin{array}{l}16,8 \\
(3,9)\end{array}$ & $\begin{array}{l}16,8 \\
(4,1)\end{array}$ & $\begin{array}{l}15,6 \\
(4,8)\end{array}$ & $\begin{array}{l}15,8 \\
(4,7)\end{array}$ & $\begin{array}{l}16,5 \\
(4,2)\end{array}$ & $\begin{array}{l}15,9 \\
(4,5)\end{array}$ & $\begin{array}{l}19,5 \\
(2,4)\end{array}$ \\
\hline CE & $\begin{array}{l}15,0 \\
(3,4)\end{array}$ & $\begin{array}{l}14, \\
(1,9)\end{array}$ & $\begin{array}{l}14,7 \\
(2,7)\end{array}$ & $\begin{array}{l}14,6 \\
(2,9)\end{array}$ & $\begin{array}{l}14,9 \\
(1,6)\end{array}$ & $\begin{array}{l}14,4 \\
(3,5)\end{array}$ & $\begin{array}{l}14,6 \\
(2,1)\end{array}$ & $\begin{array}{l}14,6 \\
(3,3)\end{array}$ & $\begin{array}{l}14,5 \\
(2,8)\end{array}$ & $\begin{array}{l}15,7 \\
(0,9)\end{array}$ \\
\hline RE & $\begin{array}{l}108,5 \\
(21,1)\end{array}$ & $\begin{array}{l}110,6 \\
(20,0)\end{array}$ & $\begin{array}{l}105,8 \\
(22,6)\end{array}$ & $\begin{array}{l}112,8 \\
(18,0)\end{array}$ & $\begin{array}{l}107,9 \\
(16,4)\end{array}$ & $\begin{array}{l}111,0 \\
(25,6)\end{array}$ & $\begin{array}{l}107,8 \\
(19,3)\end{array}$ & $\begin{array}{l}111,4 \\
(21,7)\end{array}$ & $\begin{array}{l}107,8 \\
(20,4)\end{array}$ & $\begin{array}{l}128,2 \\
(5,8)\end{array}$ \\
\hline
\end{tabular}

Nota. $*$ - $p \leq 0,05 ; * *-p \leq 0,001 ; M-$ Média; DP - Desvio Padrão; V = Vítimas; V-P = Vítimas e Perpetradoras; Z = Kolmogorov-Smirnov Z; A = Ansiedade; $\mathrm{D}=$ Depressão; LE = Acesso Limitado a Estratégias de Regulação Emocional que são Percebidas como Efetivas; NE = Não Aceitação das Emoções Negativas; FC = Falta de Consciência Emocional; CI = Capacidade para Controlar Comportamentos Impulsivos; DI = Dificuldades em Controlar Comportamento Impulsivo quando Vivencia Emoções Negativas; CE = Falta de Clareza Emocional; RE = Regulação Emocional. 
O número das participantes nos grupos foi semelhante nas dimensões negociação ( $\mathrm{G} 1, n=23$; e $\mathrm{G} 2$, $n=24)$, agressão psicológica (G1, $n=22$; e G2, $n=$ 25), agressão física (G1,n=22; e $\mathrm{G} 2, n=25)$, lesão corporal (G1, $n=24$; e G2, $n=23)$, com exceção do grupo coerção sexual (G1, $n=44$; e G2, $n=3$ ). Os resultados demonstraram que os grupos G1 e G2 não apresentaram diferenças significativas nos níveis de depressão, ansiedade, regulação emocional e suas dimensões (ver Tabela 2).

\section{Discussão}

A amostra estudada apresentou, em média, níveis leves de ansiedade e moderados de depressão. Estes resultados corroboram com os estudos de Adeodato et al. (2005) e Mozzambani et al. (2012) que encontraram associações entre violência conjugal e sintomas de ansiedade e depressão. A presença de sintomas de depressão e ansiedade podem dificultar o rompimento do ciclo de violência e a capacidade de resolução de problemas dessas mulheres. Esta experiência pode gerar efeitos negativos para autoestima e autoimagem das mulheres (Mozzambani et al., 2009).

Contudo, os níveis de ansiedade e depressão identificados nos estudos de Adeodato et al. (2005) e de Mozzambani (2012) foram mais graves. Essa diferença pode ser explicada pelo alto índice de participantes, no presente estudo, que fazia uso de psicofármacos. A medicação pode ser um fator atenuador da sintomatologia. Quase metade da amostra relatou fazer uso de psicofármacos e tal uso apresentou relação positiva com a idade. Em um estudo realizado com 360 mulheres atendidas na rede básica de saúde foram avaliados a presença de transtornos mentais e uso de psicofármacos. Dentre as variáveis associadas à presença de transtornos mentais, destacou-se o histórico de violência doméstica e $30 \%$ da amostra fazia uso de psicofármacos. Os resultados ainda indicaram a uma associação entre o uso de psicofármacos e idade, com o aumento do consumo de psicofármacos em mulheres a partir de 40 anos (Vidal, Yañez, Chaves, Yañez, Michalaros, \& Almeida, 2013). No estudo de Deeke et al. (2009), 56,7\% das mulheres em situação de violência conjugal faziam uso de medicamentos. Dessa forma, verifica-se a associação entre experiências de violência e uso de ansiolíticos ou antidepressivos como estratégia para lidar com o sofrimento psicológico. As mulheres esperam que os psicofármacos aliviem a sensação de impotência, depressão, ansiedade e outras emoções negativas decorrentes da violência (Adeodato et al., 2005; Deeke et al., 2009).

As participantes da pesquisa apresentaram níveis moderados de desregulação emocional. Indivíduos regulados emocionalmente possuem a capacidade de responder às exigências encontradas durante a vida, utilizando variadas respostas emocionais de forma flexível e aceitas socialmente. As dificuldades de regulação emocional identificadas na amostra podem auxiliar na compreensão de padrões da dinâmica da violência. As dificuldades para expressar e manejar assertivamente as emoções podem contribuir para potencializar conflitos e incitar o uso de estratégias violentas para resolver problemas conjugais (Zamir \& Lavee, 2014). Portanto, a violência conjugal pode ser vista como um resultado de padrões relacionais desadaptativos (Berzenski \& Yates, 2010).

Quando se trata de relacionamentos íntimos, a regulação emocional pode ser uma importante influência para o enfrentamento de conflitos. Dentre os fatores de risco para a perpetuação da violência, destaca-se a falta de estratégias de enfrentamento, aceitação de estratégias de enfrentamento violentas, bem como a propensão para manejo de situações conflitivas por meio da raiva reativa. Nesse sentido, a violência na relação conjugal pode estar relacionada a estratégias disfuncionais de regulação emocional (Berzenski \& Yates, 2010).

Outro resultado significativo refere-se à ausência de diferença nos níveis de ansiedade, depressão e desregulação emocional entre os grupos de mulheres que relataram sofrer agressões (G1) e de mulheres que identificaram sofrer e perpetrar agressões (G2). A violência conjugal não pode ser compreendida em uma lógica dicotômica estática (vítima $\times$ agressor). As mulheres que sofrem violência também podem se utilizar de estratégias violentas para defender-se ou lidar com situações conflitivas. A coerção sexual foi a forma de agressão menos perpetrada pelas mulheres $(n=3)$ neste estudo e, possivelmente isso ocorre pela disparidade em termos de força física para tal tipo de violência. A experiência de violência gerou níveis de depressão, ansiedade e regulação emocional semelhantes entre os grupos, indicando que esta é uma situação de risco para a saúde mental das mulheres que sofrem e também perpetram violência. A escassez de literatura sobre agressões perpetradas por mulheres em relação aos cônjuges e possíveis consequências para sua saúde indica a necessidade de investimentos em estudos nessa área (Cezario \& Lourenço, 2013; Falcke, Oliveira, Rosa, $\&$ Bentancur, 2009). 
Para compreender as consequências da violência contra a mulher, especificamente suas reações comportamentais, emocionais e sociais, torna-se necessário considerar as relações desiguais de gênero (Santos \& Moré, 2011). Em nossa sociedade, a superioridade do gênero masculino sobre o feminino, mantida culturalmente, incita a agressividade e mantém a relação de dominação do homem sobre a mulher (Caldwell et al., 2012). Nos estudos tradicionais sobre violência de gênero, as mulheres são descritas como vítima passiva da violência perpetrada pelos homens. Nesse entendimento, tais estudos referem-se à mulher vitimada como nãosujeito (identidade/ singularidade), o que suprime a possibilidade de envolver-se de forma ativa no relacionamento conjugal violento (Saffioti, 1997).

A violência não se configura como uma característica específica dos homens, podendo ser perpetrada também por mulheres, embora de forma desigual (Santos \& Izumino, 2005). A motivação das mulheres para a agressão, bem como o contexto em que a violência ocorre são qualitativamente diferentes dos homens. É provável que muitas mulheres em situação de violência conjugal utilizem a violência contra seus parceiros como uma estratégia de sobrevivência (Swan \& Snow, 2006).

\section{Considerações Finais}

As participantes deste estudo apresentaram, em média, níveis leves de ansiedade e níveis moderados de depressão e de desregulação emocional. Não foram encontradas diferenças nos níveis de ansiedade, depressão e desregulação emocional entre os grupos de mulheres que sofreram agressões (G1) e de mulheres que identificaram sofrer e perpetrar agressões (G2). Além disso, foi verificado alto índice de uso de psicofármacos, que podem ser usados como uma estratégia para diminuir o sofrimento causado pela violência e, consequentemente, pode gerar um impacto na gravidade dos sintomas avaliados.

Os resultados obtidos nesta pesquisa indicam potencial prejuízo da exposição à violência na saúde mental de mulheres nessa situação. Dessa forma, conclui-se que a avaliação psicológica pode ser útil para detectar os sintomas relacionados com a exposição à violência, tanto para as mulheres vítimas quanto para perpetradoras, visando o desenvolvimento de planos de tratamento psicológicos ajustados às demandas clínicas das mulheres nesse contexto.
O estudo apresentou algumas limitações metodológicas. Dentre elas, destaca-se o número de participantes e seleção por conveniência, que inviabiliza a generalização dos resultados. Além disso, outra limitação significativa é a ausência de um grupo de comparação (mulheres sem histórico de violência conjugal) que permitiria avaliar diferenças entre níveis de depressão, ansiedade e regulação emocional entre mulheres com e sem histórico de violência conjugal. Futuros estudos sobre as relações entre a violência conjugal, transtornos mentais e o abuso na infância devem ser realizados a fim de obter uma melhor compreensão sobre o impacto da exposição prolongada à violência para a saúde mental dos envolvidos e para auxiliar em programas de prevenção. Além disso, sugerem-se estudos futuros que busquem compreender a dinâmica e o impacto da violência sofrida e perpetrada pelas mulheres nas relações íntimas.

\section{Referências}

Adeodato, V. G., Carvalho, R. R., Siqueira, V. R., \& Souza, F. G. M. (2005). Qualidade de vida e depressão em mulheres vítimas de seus parceiros. Revista de Saúde Pública, 39(1), 108-113. doi: 10.1590/S0034-89102005000100014

American Psychiatry Association (2014). Manual diagnóstico e estatístico de transtornos mentais (DSM-5). Porto Alegre: Artmed.

Berzenski, S. R., \& Yates, T. M. (2010). A developmental process analysis of the contribution of childhood emotional abuse to relationship violence. Journal of Aggression, Maltreatment \& Trauma, 19(2), 180-203. doi: 10.1080/10926770903539474

Boeckel, M. G. (2013). Ambientes familiares tóxicos: Impactos da violência conjugal na vinculação entre mães e filhos, no reconbecimento de emoçoes e nos niveis de cortisol. Tese de Doutorado - Pós-Graduação em Psicologia, Pontifícia Universidade Católica do Rio Grande do Sul, Porto Alegre. Recuperado de http://hdl.handle.net/10923/5482

Brasil. Ministério da Saúde. (2008). Temático de prevenção de violência e cultura de paz III. Brasília, DF. Recuperado de http://pesquisa.bvsalud.org/bvsms/ resource/pt/mis-21168

Burns, E. E., Jackson, J. L., \& Harding, H. G. (2011). Child maltreatment, emotion regulation, and posttraumatic stress: The impact of emotional abuse. 
Journal of Aggression, Maltreatment \& Trauma, 19(8), 801-819. doi: 10.1080/10926771.2010.522947

Caldwell, J. E., Swan, S. C., \& Woodbrown, V. D. (2012). Gender differences in intimate partner violence outcomes. Psychology of Violence, 2(1), 42-57. doi: $10.1037 / \mathrm{a} 0026296$

Castro, R., \& Riquer, R. F. (2003). Research on violence against women in Latin America: From blind empiricism to theory without data. Cadernos de Saúde Pública, 19(1), 135-146. doi: 10.1590/ S0102-311X2003000100015

Cezario, A. C. F., \& Lourenço, L. M. (2013). Violência conjugal contra o homem: Uma análise bibliométrica. Gerais: Revista Interinstitucional de Psicologia, 6(1), 144-156.

Coutinho, J., Ribeiro, E., Ferreirinha, R., \& Dias, P. (2010). Versão portuguesa da Escala de Dificuldade de Regulação Emocional e sua relação com sintomas psicopatológicos. Revista de Psiquiatria Clinica, 37(4), 145-151. doi: 10.1590/ S0101-60832010000400001

Cunha, J. A. (2001). Manual da versão em português das Escalas Beck. São Paulo: Casa do Psicólogo.

D’Oliveira, A. F. P. L., Schraiber, L. B., França-Junior, I., Ludemir, A. B., Portella, A. P., Diniz, C. S., ... Couto, M. T. (2009). Fatores associados à violência por parceiro íntimo em mulheres brasileiras. Revista de Saúde Pública, 40, 299-310. doi: 10.1590/ S0034-89102009005000013

Deeke, L. P., Boing, A. F., Oliveira, W. F., \& Coelho, E. B. S. (2009). A dinâmica da violência doméstica: Uma análise a partir dos discursos da mulher agredida e de seu parceiro. Saúde e Sociedade, 18(2), 248-258. doi: 10.1590/S0104-12902009000200008

Dillon, G., Hussain, R., Loxton, D., \& Rahman, S. (2013). Mental and physical health and intimate partner violence against women: A review of the literature. International Journal of Family Medicine, 13. doi:10.1155/2013/313909

Falcke, D., Oliveira, D. Z., Rosa, L. W., \& Bentancur, M. (2009). Violência conjugal: Um fenômeno interacional. Contextos Clínicos, 2(2), 81-90. doi: 10.4013/ ctc.2009.22.02

Field, A. (2005). Discovering Statistics Using SPSS. 2nd Ed. London: Sage.
Garcia, L. P., Freitas, L. R. S., \& Hofelmann, D. A. (2013). Avaliação do impacto da Lei Maria da Penha sobre a mortalidade de mulheres por agressões no Brasil, 2001-2011. Epidemiologia e Serviços de Saúde, 22(3), 383-394.

Gomes, R., Minayo, M. C. S., \& Silva, C. F. R. (2005). Violência contra a mulher: Uma questão transnacional e transcultural das relações de gênero. In Brasil, Ministério da Saúde. Secretaria de Vigilância em Saúde. Impacto da violência na saúde dos brasileiros/ Ministério da Saúde, Secretaria de Vigilância em Saúde (pp. 117-140). Brasília: Ministério da Saúde.

Gorenstein, C., Pang W. Y., Argimon, I. L., \& Werlang, B. S. G. (2011). Manual do Inventário de Depressão Becke - BDI-II. São Paulo: Casa do Psicólogo.

Gratz, K. L., \& Roemer, L. (2004). Multidimensional assessment of emotion regulation and dysregulation: Development, factor structure, and initial validation of the Difficulties in Emotion Regulation Scale. Journal of Psychopathology and Behavioral Assessment, 26, 41-54. doi: 10.1023/B:JO BA.0000007455.08539.94

Gratz, K. L., Paulson, A., Jakupcak, M., \& Tull, M. T. (2009). Exploring the relationship between childhood maltreatment and intimate partner abuse: Gender differences in the mediating role of emotion dysregulation. Violence and Victims, 24(1), 68-82. doi: 10.1891/0886-6708.24.1.68

Gross, J. J. (2013). Handbook of emotion regulation. London: The Guilford Press.

Hatzenberger, R., Lima, A. P. V. R., Lobo, B., Leite, L., \& Kristensen, C. H. (2010). Transtorno de estresse pós-traumático e prejuízos cognitivos em mulheres vítimas de violência pelo parceiro íntimo. Ciências \& Cognição, 15(2), 94-110. Recuperado de http:// www.cienciasecognicao.org/revista/index.php/ cec/article/view/277/192

Krug, E. G., Dahlberg, L. L., Mercy, J. A., Zwi, A. B., \& Lozano, R. (2002). World report on violence and health. Geneva, Switzerland: World Health Organization.

Lamoglia, C. V. A., \& Minayo, M. C. S. (2009). Violência conjugal, um problema social e de saúde pública: Estudo em uma delegacia do interior do Rio de Janeiro. Ciência \& Saúde Coletiva, 14, 595-604. doi: 10.1590/S1413-81232009000200028 
Lei $n^{0}$ 11.340, de 07 de agosto de 2006. Dispõe sobre os mecanismos para coibir a violência doméstica e familiar contra a mulher. (2006). Brasil. Recuperado de http://www.planalto.gov.br/ccivil_03/_ato2004-2006/2006/lei/111340.htm

Lima, G. Q. (2010). História de vida e escolha conjugal em mulheres que sofrem violência doméstica (Dissertação de metrado). Pós-Graduação em Psicologia Clínica, Pontifícia Universidade Católica do Rio Grande do Sul, Porto Alegre. Recuperado de http://repositorio.pucrs.br/dspace/handle/10923/4788

Matud, P., Fortes, D., \& Medina, L. (2014). Eficacia de un programa de tratamiento psicológico individual para mujeres maltratadas por su pareja. Psychosocial Intervention, 23, 199-207. doi: 10.1016/j. psi.2014.06.001

Minayo, M. C. S. (2005). Violência: Um problema para a saúde dos brasileiros. Em Brasil, Ministério da Saúde. Secretaria de Vigilância em Saúde. Impacto da violência na saúde dos brasileiros / Ministério da Saúde, Secretaria de Vigilância em Saúde (pp. 9-42) Brasília: Ministério da Saúde.

Moraes, C. L., Hasselmann, M. H., \& Reichenheim, M. E. (2002). Adaptação transcultural para o português do instrumento "Revised Conflict Tactics Scales (CTS2)" utilizado para identificar violência entre casais. Caderno de Saúde Pública, 18(1), 163176. doi: 10.1590/S0102-311X2002000100017

Mozzambani, A. C. F., Ribeiro, R. L., Fuso, S. F., Fiks, J. P., \& Mello. (2009). Gravidade psicopatológica em mulheres vítimas de violência doméstica. Revista de Psiquiatria do Rio Grande do Sul, 33(1), 43-47. doi: 10.1590/S0101-81082011005000007

Organização Mundial da Saúde (OMS). (2002). Relatório mundial sobre violência e saúde. Brasilia: OMS/OPAS.

Organização Mundial de Saúde (OMS). (2014). Relatório Mundial Sobre a Prevenção da Violência 2014, São Paulo.

Organização Mundial de Saúde. Organização Pan -Americana de Saúde. La unidad de salud de la mujer de la OMS (WHD) (1998). Violencia contra la mujer: un tema de salud prioritario. Ginebra. (Sexta Sesión Plenaria, 25 de mayo de 1996. Junio 1998 - A 49-vr-6).

Robson, C. (1993). Real World Research. Oxford: Blackwells.
Rodrigues, A. P. G., \& Gondim, S. G. (2013). Expressão e regulação emocional no contexto de trabalho: Um estudo com servidores públicos. Revista de $A d$ ministração Mackenzie, 15(2), 38-65. Recuperado de www.scielo.br/pdf/ram/v15n2/v15n2a03.pdf

Saffioti, H. I. B. (1997). Violência de gênero: Lugar da práxis na construção da subjetividade. Lutas Sociais, 2, 59-79. Recuperado de http://revistas.pucsp.br/ index.php/ls/article/view/18789/13973

Santi, L. N., Nakano, A. M. S., \& Lettiere, A. (2010). Percepção de mulheres em situação de violência sobre o suporte e apoio recebido em seu contexto social. Texto \& Contexto Enfermagem, 19(3), 417424. doi: 10.1590/S0104-07072010000300002

Santos, A. C. W., \& Moré, C. L. O. O. (2011). Repercussão da violência na mulher e suas formas de enfrentamento. Paidéia, 21(49), 227-235. doi: 10.1590/S0103-863X2011000200010

Santos, C. M., \& Izumino, W. P. (2005). Violência contra as mulheres e violência de gênero: Notas sobre estudos feministas no Brasil. EIAL, Estudios Interdisciplinarios de América Latina y El Caribe, 16(1), 147-164. Recuperado de http://www.nevusp.org/ downloads/down083.pdf

Schraiber, L. B., \& D’Oliveira, A. F. P. L. (1999). Violência contra mulheres: Interfaces com a saúde. Interface Comunicação, Saúde, Educação, 3(5), 11-26. Recuperado de http://www.scielo.br/scielo.php?script=sci_arttext\&pid=S1414-32831999000200003

Schraiber, L. B., D'Oliveira, A. F. P. L., Falcão, M. T. C., \& Figueiredo, W. S. (2005). Violência dói e não é direito: A violência contra a mulher, a saúde e os direitos humanos. São Paulo: Editora UNESP.

Siegel, J. (2013). Breaking the Links in Intergenerational Violence: An Emotional Regulation Perspective. Family Process, 52, 163-178. doi: 10.1111/famp.12023

Siegel, J. P., \& Forero, R. M. (2012). Splitting and emotional regulation in partner violence. Clinical Social Work Journal, 40, 224-230. doi: 10.1007/ s10615-011-0352-3

Stith, S. M., \& McCollum, E. E. (2011). Conjoint treatment of couples who have experienced intimate partner violence. Aggression and Violent Behavior, 16, 312-318. doi:10.1016/j.avb.2011.04.012

Straus, M. A., Hamby, S. L., Buncy-McCoy, S., \& Sugarman, D. B. (1996). The revised Conflict Tactics 
Scale (CTS2): Development and preliminary psychometric data. Journal of Family Issues, 17(3), 283-316.

Tavares, F. A., \& Pereira, G. C. (2007). Reflexos da dor: Contextualizando a situação das mulheres em situação de violência doméstica. Revista Textos \& Contextos, 6(2), 410-424. Recuperado de http:// revistaseletronicas.pucrs.br/face/ojs/index.php/ article/view/2318/3255

Vidal, C. E. L., Yañez, B. F. P., Chaves, C. V. S., Yañez, C. F. P., Michalaros, I. A., \& Almeida, L. A. S. (2013). Transtornos mentais comuns e uso de psicofármacos em mulheres. Caderno de Saúde Coletiva, 21(4), 457-464.

Vieira, E. M., Perdona, G. S. C., \& Santos, M. A. (2011). Fatores associados à violência física por parceiro íntimo em usuárias de serviços de saúde. Revista de Saúde Pública, 45(4), 730-737. doi: 10.1590/S0034-89102011005000034

Waiselfisz, J. J. (2015). Mapa da Violência 2015: Homicídio de mulheres no Brasil. Brasilia, DF.

Zaleski, M., Pinsky, I., Laranjeira, R., Ramisetty-Mikler, S., \& Caetano, R. (2010). Violência entre parceiros íntimos e consumo de álcool. Revista de Saúde Pública, 44(1), 53-59. doi: 10.1590/ S0034-89102010000100006

Zamir, O., \& Lavee, Y. (2014). Emotional Regulation and Revictimization in Women's Intimate Relationships. Journal of Interpersonal Violence, 31(1), 147-62. doi: $0.1177 / 0886260514555125$

Recebido em: 09/01/2017

Reformulado em: 15/05/2017

Aprovado em: 04/08/2017

Sobre as autoras:

Me. Natália Zancan é psicóloga clínica, mestre em Psicologia Clínica pela Pontifícia Universidade Católica do Rio Grande do Sul (PUCRS) e especialista em Terapia Cognitivo-Comportamental (IMED), com formação em Terapia Comportamental Dialética (Behavioral Tech/EUA e The Linehan Institute/EUA).

E-mail: natalia.zancan@yahoo.com

Dra. Luísa Fernanda Habigzang é doutora em Psicologia, professora do Curso de Graduação e do Programa de Pós-Graduação em Psicologia da Pontifícia Universidade Católica do Rio Grande do Sul (PUCRS) e coordenadora do Grupo de Pesquisa Violência, Vulnerabilidade e Intervenções Clínicas (GPEVVIC).

E-mail: luisa.habigzang@pucrs.br

Contato com as autoras:

GPeVVIC - Grupo de Pesquisa Violência, Vulnerabilidade e Intervenções Clínicas

Pontifícia Universidade Católica do Rio Grande do Sul

Av. Ipiranga, 6681, Prédio 11, $9^{\circ}$ andar, sala 924

Porto Alegre-RS, Brasil

CEP: 90619-900

Psico-USF, Bragança Paulista, v. 23, n. 2, p. 253-265, abr./jun. 2018 